AIP $\mid \begin{aligned} & \text { Jumalot } \\ & \text { Applied Physics }\end{aligned}$

\title{
Carrier capture dynamics of single InGaAs/GaAs quantum-dot layers
}

K. N. Chauhan, D. M. Riffe, E. A. Everett, D. J. Kim, H. Yang et al.

Citation: J. Appl. Phys. 113, 203710 (2013); doi: 10.1063/1.4808337

View online: http://dx.doi.org/10.1063/1.4808337

View Table of Contents: http://jap.aip.org/resource/1/JAPIAU/v113/i20

Published by the AIP Publishing LLC.

\section{Additional information on J. Appl. Phys.}

Journal Homepage: http://jap.aip.org/

Journal Information: http://jap.aip.org/about/about_the_journal

Top downloads: http://jap.aip.org/features/most_downloaded

Information for Authors: http://jap.aip.org/authors

\section{ADVERTISEMENT}

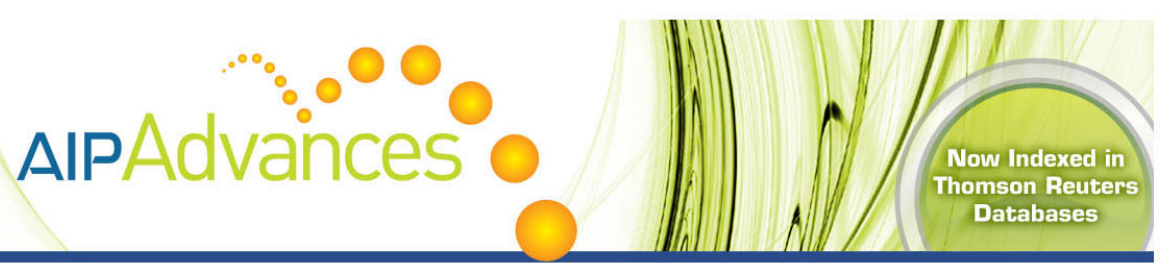

\section{Explore AIP's open access journal: Rapid publication \\ - Article-level metrics \\ - Post-publication rating and commenting}




\title{
Carrier capture dynamics of single InGaAs/GaAs quantum-dot layers
}

\author{
K. N. Chauhan, ${ }^{1}$ D. M. Riffe, ${ }^{1, a)}$ E. A. Everett, ${ }^{1}$ D. J. Kim, ${ }^{1}$ H. Yang, ${ }^{1, b)}$ and F. K. Shen ${ }^{2}$ \\ ${ }^{1}$ Physics Department, Utah State University, Logan, Utah 84322-4415, USA \\ ${ }^{2}$ Center for Surface Analysis and Applications, Utah State University, Logan, Utah 84322-4415, USA
}

(Received 15 March 2013; accepted 16 May 2013; published online 31 May 2013)

\begin{abstract}
Using $800 \mathrm{~nm}, 25$-fs pulses from a mode locked $\mathrm{Ti}_{\mathrm{Al}} \mathrm{Al}_{2} \mathrm{O}_{3}$ laser, we have measured the ultrafast optical reflectivity of MBE-grown, single-layer $\mathrm{In}_{0.4} \mathrm{Ga}_{0.6} \mathrm{As} / \mathrm{GaAs}$ quantum-dot (QD) samples. The QDs are formed via two-stage Stranski-Krastanov growth: following initial InGaAs deposition at a relatively low temperature, self assembly of the QDs occurs during a subsequent higher temperature anneal. The capture times for free carriers excited in the surrounding GaAs (barrier layer) are as short as $140 \mathrm{fs}$, indicating capture efficiencies for the InGaAs quantum layer approaching 1. The capture rates are positively correlated with initial InGaAs thickness and annealing temperature. With increasing excited carrier density, the capture rate decreases; this slowing of the dynamics is attributed to Pauli state blocking within the InGaAs quantum layer. (C) 2013 AIP Publishing LLC. [http://dx.doi.org/10.1063/1.4808337]
\end{abstract}

\section{INTRODUCTION}

Due to both potential and realized photonics applications, carrier dynamics in self-assembled $\mathrm{InGaAs} / \mathrm{GaAs}$ quantum dot (QD) systems have been the subject of numerous investigations. In the time domain, the main tools for these investigations have been time resolved photoluminescence (PL) and time resolved pump-probe transmission measurements. (See, for example, Refs. 1-3.) In a typical experiment, carriers in the surrounding GaAs barrier layers are initially excited. These carriers can then become captured by the InGaAs quantum layer (QL) [comprising the selfassembled QDs on top of a wetting layer (WL)], relax through states within the QL, and then recombine, often radiatively. Depending upon the details of the sample geometry, transport of the excited carriers within the barrier layer may be necessary before trapping by the QL can occur. These investigations have primarily focused on the relaxation of carriers within the QL system. The dynamics immediately after initial excitation-transport and capture-have been less thoroughly investigated.

The QD structures studied here are self assembled using a modified Stranski-Krastanov (SK) growth process (reviewed below). ${ }^{4}$ Key to this process is the formation of QDs from an atomically flat (but strained) InGaAs layer during a high-temperature anneal of the sample. With this modified technique, the QD morphology can be controlled not only via the amount of deposited InGaAs but also through the annealing time and temperature. Various structures, including separated QDs, QD chains, and quantum dashes, have been formed using this process. ${ }^{4,5}$

With the ability to prepare distinct QD structures comes the potential to systematically investigate connections between QL morphology and carrier dynamics. In this paper,

\footnotetext{
${ }^{\text {a) }}$ Author to whom correspondence should be addressed; Electronic mail: mark.riffe@usu.edu

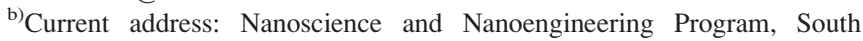
Dakota School of Mines and Technology, Rapid City, South Dakota 57701, USA.
}

we present results on carrier capture by the QL in three InGaAs/GaAs QD samples, all grown using modified SK self assembly. Our results show that the dynamics indeed depend upon the morphology: a thicker QL and a higher density and/or size of the dots results in faster capture by the quantum layer. Together with a diffusion model for carrier transport, our results further suggest that transport near the QL proceeds via ambipolar diffusion. Our experiments, carried out at relatively high excitation levels, also reveal the impact that state blocking has upon carrier dynamics in these QL systems.

\section{SAMPLE AND EXPERIMENTAL DETAILS}

The modified Stranski-Krastanov growth process has been previously described in detail; ${ }^{4}$ we briefly review it here. After initial processing of the GaAs(100) substrate, a GaAs buffer layer (1300 to $2300 \mathrm{~nm}$ ) is grown with the substrate held at $\sim 590^{\circ} \mathrm{C}$. The sample is then cooled to a growth temperature $\left(T_{G}\right)$ of 360 or $370^{\circ} \mathrm{C}$ and approximately 10 or 15 monolayers (ML) of $\mathrm{In}_{0.4} \mathrm{Ga}_{0.6}$ As are grown. At this relatively low temperature, the InGaAs layer remains atomically flat. The InGaAs QDs are then formed by heating the sample at a rate of $20^{\circ} \mathrm{C} / \mathrm{min}$ under As flux to an annealing temperature $\left(T_{A}\right)$ in the range 470 to $490^{\circ} \mathrm{C} ; T_{A}$ is maintained for $120 \mathrm{~s}$. The samples are then capped with $\sim 10 \mathrm{~nm}$ of GaAs before being removed from the growth chamber. The specific growth parameters for the samples studied here are shown in Table I. Reflection high-energy electron diffraction (RHEED) is used to determine the thickness of the initial InGaAs layer and also to monitor the formation of the QDs. As noted in Table I, the RHEED observations clearly indicated the formation of QDs on samples B and C but not on sample A.

The QD morphology of other $\sim 10$ ML samples grown in the same manner, but not capped, has been previously investigated with in situ scanning tunneling microscopy (STM). ${ }^{4}$ The STM measurements reveal a QD density between 1.2 and $2.3 \times 10^{11} \mathrm{~cm}^{-2}$, with the typical QD 
having a base of $\sim 25 \mathrm{~nm}$ and a height of $\sim 8 \mathrm{~nm}$. The morphology of the dots is sensitive to both the InGaAs growth and annealing temperatures. Notably, at higher annealing temperatures, the dots align themselves in chains which, on average, lie along the $[1 \overline{1} 0]$ direction.

We have assessed the morphology of the capped samples studied here with ex situ atomic force microscopy (AFM); images of the samples are shown in Fig. 1. Although the thin GaAs cap obscures the finer features associated with the underlying QD structure, the AFM images clearly distinguish differences in QD morphology among the samples. Samples B and C exhibit structure that is most similar to the uncapped samples previous studied by STM: the dots are organized into chains, and the lateral density of the chains is similar to that of the uncapped samples. Conversely, the AFM image of sample A shows only a very low density $\left(\sim 10^{9} \mathrm{~cm}^{-2}\right)$ of features that can be associated with any formation of QDs; this sparseness of QDs is consistent with the dots not being observed with RHEED.

Low-temperature $(3.6 \mathrm{~K})$ photoluminescence data from (other pieces of) these samples have been previously obtained. ${ }^{6}$ There are several features common to the PL spectra from all three samples: (i) a strong peak between 1040 and $1090 \mathrm{~nm}$, (ii) a much weaker peak centered at $920 \mathrm{~nm}$, and (iii) a tail that extends to $\sim 1450 \mathrm{~nm}$. In addition, PL spectra from sample A exhibit a $980 \mathrm{~nm}$ peak. Based on comparisons with previously published luminescence spectra from other $\mathrm{In}_{0.4} \mathrm{Ga}_{0.6} \mathrm{As} / \mathrm{GaAs}$ samples, ${ }^{7-13}$ we assign the $1000-1100 \mathrm{~nm}$ peak to ground-state (GS) emission from the dots and the 920 peak to emission from the InGaAs wetting layer (WL) that remains below the dots after the high-temperature anneal. The PL wavelength of $920 \mathrm{~nm}$ from the WL indicates a WL thickness of $6 \mathrm{ML},{ }^{14,15}$ which is equal to the critical thickness for $\mathrm{In}_{0.4} \mathrm{Ga}_{0.6} \mathrm{As} / \mathrm{GaAs} .{ }^{16}$ Also consistent with previously published PL measurements, ${ }^{14,15}$ the wavelength of $980 \mathrm{~nm}$ from sample A is assigned to emission from regions of the $\mathrm{InGaAs}$ layer that did not form QDs, and so retain their original $\sim 10 \mathrm{ML}$ thickness. The longer wavelength tail to $1450 \mathrm{~nm}$ is assigned to defect states in the vicinity of the InGaAs quantum layer (QL); these are likely interface states between the InGaAs and the GaAs cap. We have fit the QD GS peak to obtain both the peak position $\lambda_{0}$ and width $\delta \lambda$; the results of this analysis are shown in Table I.

The experimental setup for the ultrafast reflectivity measurements is similar to that for previous measurements on $\mathrm{Si}^{17}{ }^{17}$ In the present experiment near-Gaussian pulses from a Ti:sapphire oscillator ${ }^{18}(800 \mathrm{~nm}, 25 \mathrm{fs}, 1.1 \mathrm{~nJ})$ are split into

TABLE I. Sample growth parameters. Long sample designations (030907-1, e.g.) are for cross referencing to PL measurements. ${ }^{6}$ Short designations (A, e.g.) are for internal reference in this paper. Also indicated are PL peak position $\lambda_{0}$ and line width $\delta \lambda$ for ground-state QD emission.

\begin{tabular}{lcccccr}
\hline \hline Sample & $\begin{array}{c}\text { InGaAs } \\
(\mathrm{ML})\end{array}$ & $\begin{array}{c}T_{G} \\
\left({ }^{\circ} \mathrm{C}\right)\end{array}$ & $\begin{array}{c}T_{A} \\
\left({ }^{\circ} \mathrm{C}\right)\end{array}$ & $\begin{array}{c}\text { QDs via } \\
\text { RHEED }\end{array}$ & $\begin{array}{c}\lambda_{0} \\
(\mathrm{~nm})\end{array}$ & $\begin{array}{c}\delta \lambda \\
(\mathrm{nm})\end{array}$ \\
\hline $030907-1(\mathrm{~A})$ & 9.6 & 370 & 470 & No & 1080 & 110 \\
$030907-2(\mathrm{~B})$ & 9.5 & 360 & 480 & Yes & 1042 & 35 \\
$030607-2(\mathrm{C})$ & 14.6 & 370 & 490 & Yes & 1085 & 55 \\
\hline
\end{tabular}
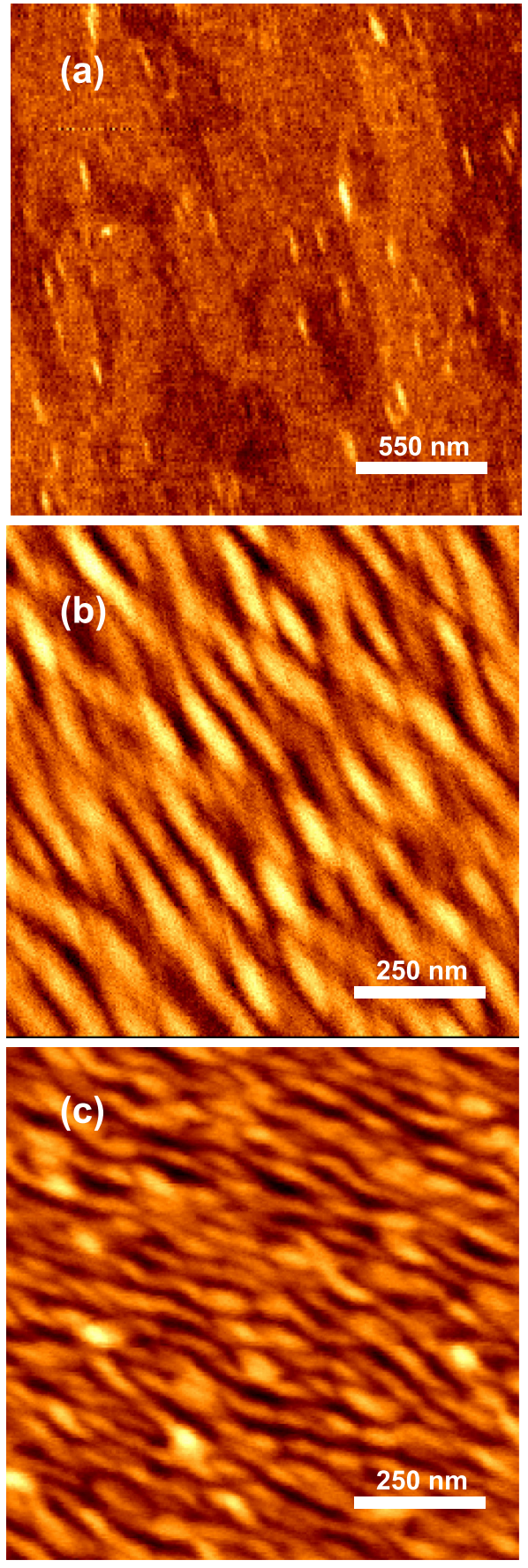

FIG. 1. AFM images of the three samples in this study. Samples A, B, and C are shown in (a), (b), and (c), respectively. Image sizes are is $2.2 \times 2.2 \mu \mathrm{m}^{2}$ for (a) and $1 \times 1 \mu \mathrm{m}^{2}$ for (b) and (c).

a pump beam (at normal incidence) and an $s$-polarized probe beam (angle of incidence $=45^{\circ}$ ). The pump beam is chopped, and changes in the probe-beam reflectivity induced by the pump beam are measured as a function of time delay between the pump and probe pulses. The pump-pulse fluence is varied between $\sim 0.006$ and $\sim 0.3 \mathrm{~mJ} / \mathrm{cm}^{2}$ using neutral density filters. Based upon the pump-pulse fluence and accounting for saturation of the excited carriers at higher fluences, ${ }^{19}$ we calculate the near-surface excited carrier density to range from $2.5 \times 10^{17} \mathrm{~cm}^{-3}$ to $4.0 \times 10^{18} \mathrm{~cm}^{-3}$ in our experiments. 


\section{RESULTS AND ANALYSIS}

Typical reflectivity data are presented in Fig. 2(a), which shows data from all three samples at two different laser intensities. For all of the samples, the initial reflectivity change is positive. For samples A and B, this is followed by a monotonic decay back toward the initial reflectivity. For sample $\mathrm{C}$, the change in reflectivity becomes slightly negative before again becoming slightly positive. Even by $120 \mathrm{ps}$ (our maximum time delay), the reflectivity of all of the samples has not yet fully recovered to its initial value. Given that PL lifetimes are typically several hundred ps for similar QD systems, ${ }^{20,21}$ a lack of total recovery in the reflectivity even by 120 ps is not surprising. Referring to Table I and Fig. 2(a) (and noting that samples A and B have essentially identical amounts of deposited InGaAs), we see that the short-time decay rate is positively correlated with both annealing temperature $T_{A}$ and the initial amount of InGaAs deposited and negatively correlated with the level of carrier excitation.

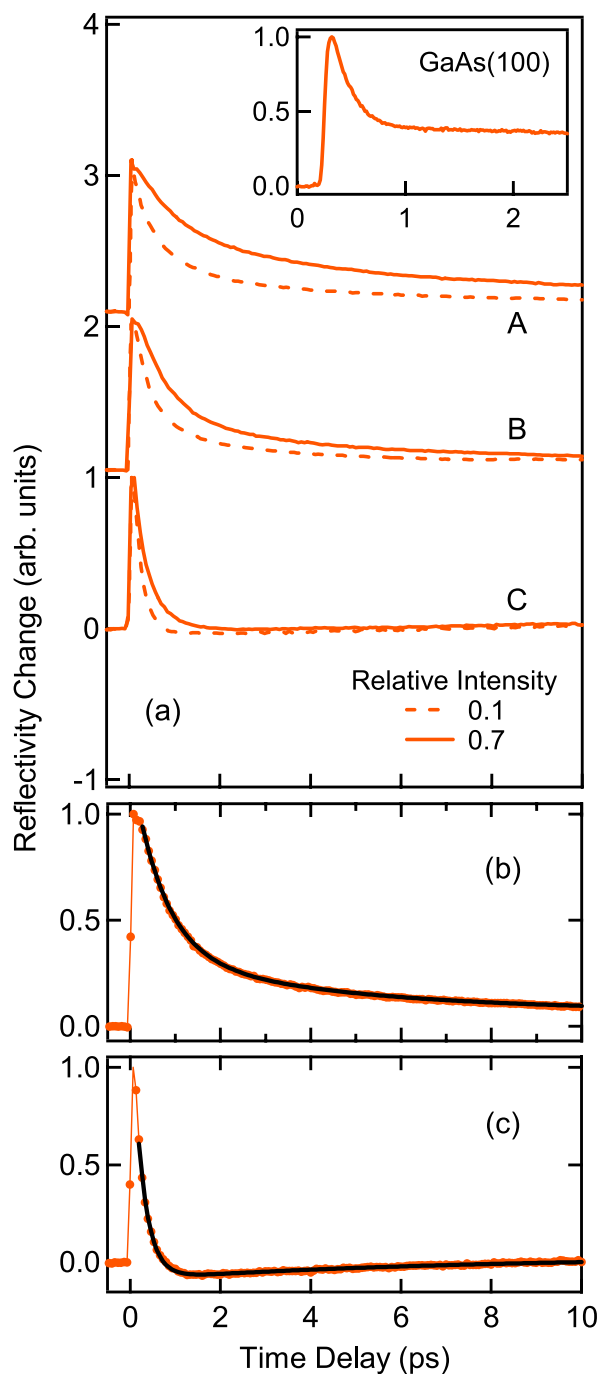

FIG. 2. Reflectivity data vs time delay. Data from samples A, B, and C (as indicated) for two different pump intensities (values are relative to maximum intensity) are shown in (a). Data for A and B are vertically shifted for clarity. For comparison, reflectivity from $\mathrm{GaAs}(100)$ is shown in the inset of (a). Fits to reflectivity data are illustrated in (b) and (c) for samples B and C at a relative laser intensity of 0.7 and 0.34 , respectively.
In order to quantitatively determine time constants associated with the reflectivity decay, we have analyzed the data using decaying exponential functions. Immediately after the reflectivity maximum, the signal exhibits complexity that is not simply modeled. This is possibly related to the complex quantum-kinetic nature of GaAs carrier dynamics at the shortest time scales. ${ }^{22,23}$ However, after a brief time ( $\sim 250 \mathrm{fs}$ ) and up to at least several ps, the data can be described by a sum of two decaying exponentials (plus a nonzero background). The fitting reveals a dominant, positive-amplitude, faster-decay component for each reflectivity curve. For samples A and B, the secondary, slowerdecay component also has a positive amplitude. For sample $\mathrm{C}$, the secondary component has a negative amplitude. Typical fits of the reflectivity curves are illustrated in parts (b) and (c) of Fig. 2.

In Fig. 3, we plot the decay time $\tau_{F}$ of the faster component as a function of laser intensity for the three samples. As the graph indicates, at the lowest laser intensity in our study $\tau_{F} \approx 140$ fs for sample $\mathrm{C}$ while $\tau_{F} \approx 280$ fs for samples $\mathrm{A}$ and $\mathrm{B}$. The figure shows for all three samples that $\tau_{F}$ monotonically increases with increasing laser intensity.

The slower-component relaxation time $\tau_{S}$ for samples A and $\mathrm{B}$ is also correlated with the pump-laser intensity. For sample $\mathrm{B}, \tau_{S}$ increases from $\sim 2.5 \mathrm{ps}$ at the lowest laser intensities to $\sim 3.5 \mathrm{ps}$ at maximum intensity. Similarly, for sample A $\tau_{S}$ varies from $\sim 3$ ps to $\sim 6$ ps as the intensity is increased. For sample $\mathrm{C}$, the longer time constant is $\sim 9 \mathrm{ps}$; its variation versus excitation density is negligible.

\section{DISCUSSION}

\section{A. Assignment of decay times}

In the measurements on the QD samples, the predominant effect of the pump pulse is to excite carriers from the valence band $(\mathrm{VB})$ to the conduction band $(\mathrm{CB})$ in the GaAs

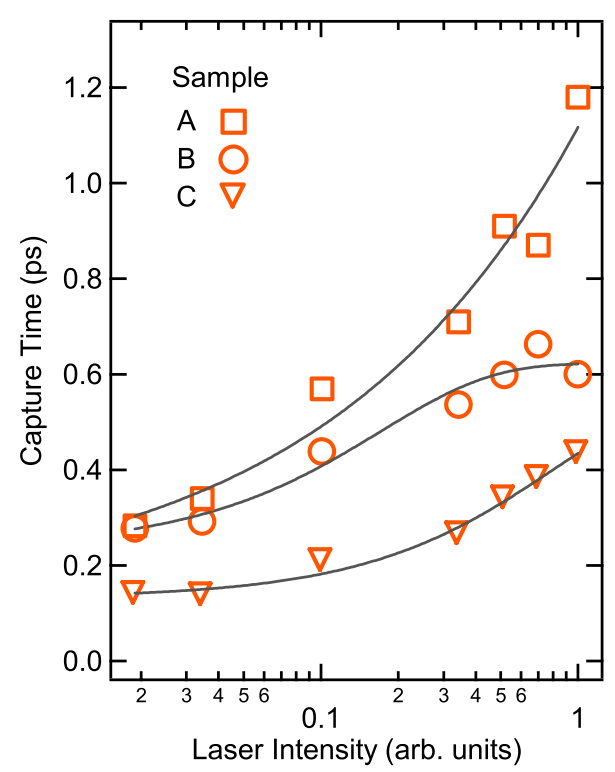

FIG. 3. Fast capture time $\tau_{F}$ vs laser intensity for samples A, B, and C. Symbols are results of fitting the reflectivity data. Solid lines are guides to the eye. 
that surrounds the InGaAs QL; in order to understand the ultrafast reflectivity from these samples, it is instructive to first review carrier dynamics in GaAs and then consider the reflectivity of a standard GaAs(100) sample. Because reflectivity data at $800 \mathrm{~nm}$ are most sensitive to the $\mathrm{CB}$ electrons (as opposed to VB holes), ${ }^{24}$ we concentrate on the electron dynamics. The pump pulse initially creates a CB electron distribution that can be characterized as having a degree of (quantum) coherence, ${ }^{25,26}$ both anisotropic and isotropic momentum-space components, ${ }^{27,28}$ and a nonthermal energy distribution. ${ }^{2,23,29,30}$ Carrier-carrier and carrier-phonon scattering relaxes these components in several, approximately sequential, ways. (i) On a time scale of a few 10 s of femtoseconds the coherence disappears and the anisotropic component relaxes, resulting in an isotropic, incoherent distribution in momentum space. ${ }^{25-27}$ (ii) On a time scale of 100 to $200 \mathrm{fs}$, the nonthermal energy distribution becomes thermalized, but still hot. ${ }^{29-31}$ (iii) This distribution then cools close to the initial sample temperature on a time scale of a few ps. $^{32}$ (iv) On a much longer time scale, the excited carriers eventually recombine across the gap, producing a fully equilibrated state.

Reflectivity data from a standard GaAs(100) sample are shown in the inset of Fig. 2(a). As with the QD samples, the initial reflectivity change is positive. For $\operatorname{GaAs}(100)$, the reflectivity then rapidly decays with a time constant of $\sim 150 \mathrm{fs}$. However, unlike the QD samples, the baseline for the fast decay is close to half of the initial reflectivity change. This baseline itself slowly decays (with a time constant of $\sim 20 \mathrm{ps}$ ) back to the initial reflectivity value. Also in contrast to the QD-sample data, the rate associated with the fast decay is (nearly) independent of the excitation intensity. Based on the results of GaAs carrier-dynamics studies, the 150 fs time constant can be associated with intracarrier thermalization of the initially excited electron distribution, while the 20 ps time constant can be associated with carrier recombination, most likely through surface recombination involving defects at the $\mathrm{GaAs}(100)$ surface.

We now consider the reflectivity of the QD samples. Because the initial reflectivity change of the QD samples is positive with a magnitude comparable to that of $\operatorname{GaAs}(100)$, we associate most of the reflectivity change in these samples with the excited electron population in the GaAs that surrounds the InGaAs QL. However, unlike the reflectivity data from $\operatorname{GaAs}(100)$, we cannot assign the fastest decay to intracarrier thermalization. The reasons for this are (i) the fast decay time varies significantly from sample to sample ( $\sim 140$ fs for sample $\mathrm{C}$ to $\sim 280$ fs for samples A and B at the lowest intensity, (ii) the decay time is a strong function of exciting laser intensity, and (iii) the baseline for the initial decay is much closer to zero than for $\operatorname{GaAs}(100)$. Because of these differences, and because the decay systematically varies with annealing temperature $T_{A}$ and InGaAs growth thickness, we instead assign the decay to electron capture by the InGaAs QL.

As supported by results from a one-dimensional (1D) diffusion model (discussed below), we assign the faster decay time $\left(\tau_{F}\right)$ to the capture of electrons that are initially in the vicinity of the InGaAs QL. For samples A and B, we assign the slower decay time $\left(\tau_{S}\right)$ to the capture of electrons that must diffuse into the region near the QL before becoming trapped, although there may also be a contribution to this time constant from intra-QL relaxation. Because the secondary-component amplitude for sample $\mathrm{C}$ is negative, it is unlikely to be associated with trapping; we thus assign this relaxation time to carrier relaxation within the QL.

The significance of the fast capture times can be assessed by a simple estimation of carrier transport in the vicinity of the QL. Because the reflectivity changes are most sensitive to changes in the sample index of refraction within an observation depth $d_{o b s}=\lambda /(4 \pi n) \approx 20 \mathrm{~nm}$ of the surface ( $n$ is the GaAs index of refraction), ${ }^{17}$ we first consider the transport and capture of carriers that lie within $\pm 10 \mathrm{~nm}$ of the InGaAs QL. In our experiment, the thermalized (but still hot) electrons have a temperature of $\sim 750 \mathrm{~K},{ }^{33}$ which results in an average thermal-velocity component (perpendicular to the surface) of $\sim 3 \times 10^{7} \mathrm{~cm} / \mathrm{s}$. Thus, within $(10 \mathrm{~nm}) /\left(3 \times 10^{7} \mathrm{~cm} / \mathrm{s}\right)=33$ fs about half of the electrons within $\pm 10 \mathrm{~nm}$ of the QL have interacted with the QL. This result suggests that electron capture by sample $\mathrm{C}$ ( $\tau_{F}=140 \mathrm{fs}$ at the lowest intensities) is quite efficient.

\section{B. 1D diffusion model}

To gain further insight into the transport and capture process, we utilize a $1 \mathrm{D}$ diffusion model for the carrier density $N\left(z, t_{D}\right)\left(z=\right.$ distance into the sample, $t_{D}=$ time delay $){ }^{34}$ To keep the model as simple as possible, we assume ambipolar diffusion, which is expected to be valid as long as electron and hole capture rates are not drastically different. Because (i) ambipolar diffusion is dominated by the holes and (ii) the excited hole temperature is close to RT, we use the RT ambipolar diffusion coefficient $D_{a}=20 \mathrm{~cm}^{2} / \mathrm{s}^{35}$ The fate of a carrier incident on the QL is described using probabilities for capture, transmission, and reflection $(c, t$, and $r$, respectively; $1=c+t+r$ ). These probabilities enter the model via capture and transmission velocities, which are related to the probabilities via $v_{t}=v_{R} t$ and $v_{c}=v_{R} c$, where $v_{R}=\sqrt{k_{B} T / 2 \pi m^{*}} \approx 1 \times 10^{7} \mathrm{~cm} / \mathrm{s}$ is the Richardson velocity ( $m^{*}$ is the carrier effective mass). ${ }^{36}$ Because recombination at the native-oxide surface of our $\operatorname{GaAs}(100)$ sample occurs on a timescale $\geq 20 \mathrm{ps}$, we simply assume that the carriers are perfectly reflected from the native-oxide surface of the QD samples.

Before presenting results obtained with the diffusion model, we acknowledge several potential pitfalls associated with a 1D diffusion model for describing transport in our QD samples. First, any diffusion model assumes that the carriers are described by a thermal distribution. This assumption is reasonable in the present case because intracarrier thermalization occurs within $\sim 150$ fs. Second, the carrier mean-free path should be significantly smaller than the cap-layer thickness of $10 \mathrm{~nm}$. The $z$-direction mean-free path is $l_{z} \approx 2 v_{R} \tau_{m}$, where $\tau_{m}$ is the momentum relaxation time of the carriers. For a carrier density of $2 \times 10^{17} \mathrm{~cm}^{-2}, \tau_{m}=40 \mathrm{fs},{ }^{27}$ which yields $l_{z}=8 \mathrm{~nm}$, closer to the cap-layer thickness than is ideal for modeling the transport strictly as diffusion. Third, it is clear from the AFM images in Fig. 1 that important lateral 
length scales for the cap are similar to its thickness, indicating that a $3 \mathrm{D}$ model is probably more appropriate. Given these last two issues, we expect any parameters extracted from the diffusion-model analysis to only be approximate in value. However, as we see below, with reasonable parameter values the 1D diffusion model does produce an approximately bi-exponential decay (in time) of the carrier density near the surface of the sample, consistent with the reflectivity data.

Using the model, we have calculated $N\left(z, t_{D}\right)$ using the capture and transmission probabilities ( $c$ and $t$, respectively) as free parameters. In the experiment carrier excitation happens within several tens of fs, and so for simplicity the model is given an initial (excited) carrier density $N(z, 0)=N_{0} \exp (-z / \delta)$, where $N_{0}$ is the initial carrier density at the sample surface and $\delta=670 \mathrm{~nm}$ is the penetration depth of $800 \mathrm{~nm}$ light in GaAs. ${ }^{37}$ This is the 0-ps curve shown in Fig. 4(a). As is observed for sample C, we are able

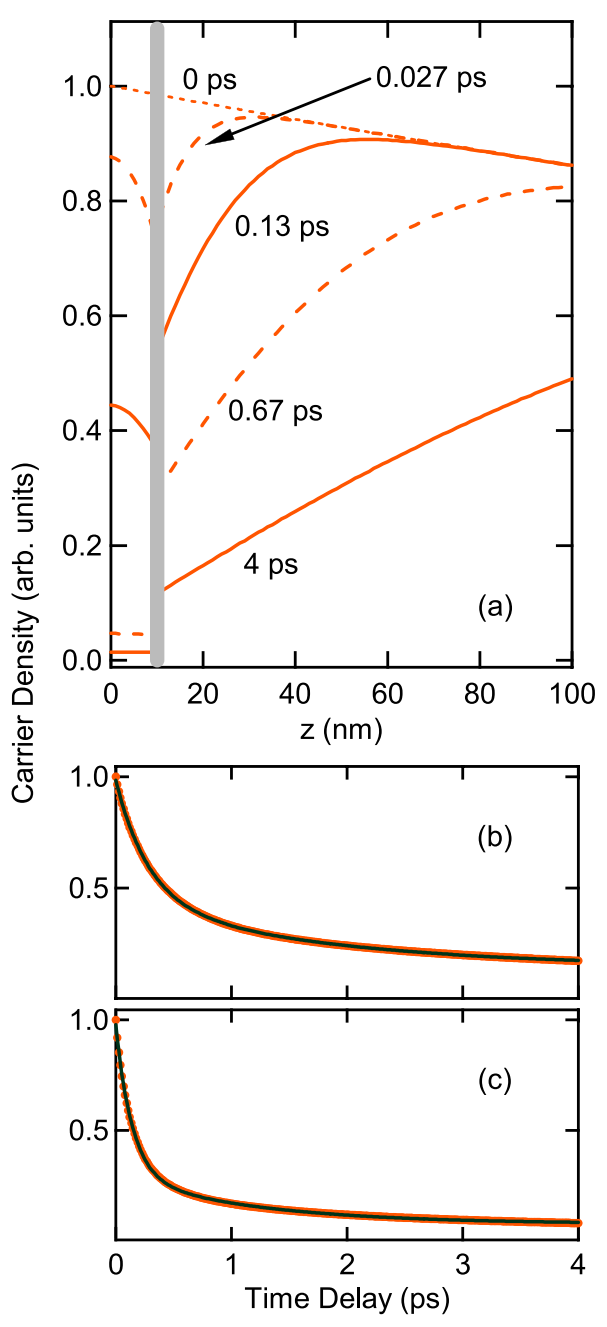

FIG. 4. Results of 1D diffusion model. (a) Carrier density $N\left(z, t_{D}\right)$ vs distance $z$ from sample surface at 5 different time delays $t_{D}$. The vertical line indicates location of QL. For this panel $t=0.1$ and $c=0.77$, which produces a fast decay similar to that of sample $\mathrm{C}$ at low excitation levels. (b) Nearsurface $(0-20 \mathrm{~nm})$ carrier density vs time delay for $c=0.3$ and $t=0.1$, which gives decay similar to that of samples $\mathrm{A}$ and $\mathrm{B}$ at low excitation level. Points are results of model; solid line is bi-exponential fit with decay times of 0.28 and $1.6 \mathrm{ps}$. (c) Near-surface carrier density vs time delay for $c=0.77$ and $t=0.1$, as in (a). Solid-line fit has decay times of 0.14 and $1.1 \mathrm{ps}$. to obtain a fast decay time of 0.14 ps for the near-surface carrier density for $c$ varying between 0.8 and 0.7 and $t$ concomitantly varying between 0 and 0.3 . In parts (a) and (c) of Fig. 4 we show results for the combination $c=0.77$ and $t=0.1$. The curves in Fig. 4(a) illustrate the carrier density in the GaAs barrier layer for several times between 0 and $4 \mathrm{ps,}$ while part (c) shows the (normalized) carrier density averaged over the first $20 \mathrm{~nm}$ of the sample. A fit to the results in (c) indeed shows that the calculated decay is well approximated by a bi-exponential model with time constants of 0.14 and 1.2 ps. Part (b) of the figure illustrates model results for $c=0.3$ and $t=0.1$, which is well fit using time constants of 0.28 and 1.8 ps. This fast time constant matches the experimental result for samples A and B at low laser intensity. As expected, slower decay times (appropriate to higher excitations levels) correspond to even smaller capture probabilities. For example, an initial capture time of 1 ps can be obtained with $c$ and $t$ values of 0.05 and 0.1 , respectively.

\section{Capture processes}

Carrier capture by the QL can proceed via either carrierphonon (cp) or carrier-carrier (cc) (i.e., Auger) scattering. For small carrier densities capture proceeds via cp scattering, but as the carrier density increases cc scattering becomes dominant, owing to the density dependence of the cc scattering rate. At our relatively high excitation densities (see details below), cc scattering is believed to be the primary capture mechanism. ${ }^{38}$ We thus might expect to see the capture time $\tau_{F}$ decrease with increasing excitation level. However, we observe just the opposite, and so another process must be responsible for the behavior of $\tau_{F}$ with increasing carrier density.

We ascribe the increase in $\tau_{F}$ with excitation level to Pauli state blocking within the quantum layer. ${ }^{39,40}$ This assessment comes from an estimation of the number of carriers captured (within the first several ps). First, at the lowest laser fluence $\left(\sim 0.006 \mathrm{~mJ} / \mathrm{cm}^{2}\right)$ the initial near-surface carrier density $N_{0}=2.5 \times 10^{17} \mathrm{~cm}^{-3}$ corresponds to an areal density $N_{0} \delta=1.7 \times 10^{13} \mathrm{~cm}^{-2}$. From the diffusion model with parameters appropriate to sample $\mathrm{C}$ at the lowest laser intensity ( $c=0.77$ and $t=0.1$, for example), we find that within $4 \mathrm{ps}$ the carrier density captured by the InGaAs QL is $\sim 2 \times 10^{12} \mathrm{~cm}^{-2}$. This corresponds to $\sim 10$ carriers/QD. At the highest intensities where the excited carrier density is $4.0 \times 10^{18} \mathrm{~cm}^{-3}$ and the capture time is between 0.4 and $1 \mathrm{ps}$, we calculate that on the order of 100 carriers/QD are captured by the QL within the first 4 ps. Such high carrier densities inhibit capture and relaxation within the QL via state blocking, and so it is no surprise that capture times increase with laser excitation. Further evidence that blocking is important comes from the second capture time $\tau_{S}$ for samples $\mathrm{A}$ and $\mathrm{B}$, which also increases with laser intensity. In addition, the experimental values of $\tau_{S}$ are somewhat longer that those deduced from the diffusion model; this is also consistent with state blocking decreasing the capture probability $c$ as carriers become trapped by the QL.

Comparisons of the $\tau_{F}$ curves vs laser intensity in Fig. 3 illuminates details of the impact that QL morphology has on 
the carrier dynamics. We first consider samples B and C, which had initial InGaAs-layers thicknesses of 9.5 and 14.6 ML, respectively. As evidenced from the AFM images and PL data, both samples comprise a high density of QDs sitting on top of a 6 ML WL, but because sample C contains a larger amount of InGaAs, we can surmise that the QDs on sample $\mathrm{C}$ are larger and/or more denser than those on sample B. A comparison of the ground-state QD PL from samples B and $\mathrm{C}$ (see Table I), which occurs at slightly longer wavelength $\left(\lambda_{0}\right)$ for sample $\mathrm{C}$, suggests that the QDs on sample C are indeed larger than those on sample B. That the lowintensity values of $\tau_{F}$ are smaller for sample $\mathrm{C}$ thus suggests direct capture (from the GaAs barrier) by the QDs occurs in addition to capture via the WL. A comparison of $\tau_{F}$ for samples A and B is also enlightening. Recall that samples A and B contain essentially equal amounts of InGaAs, but substantially fewer QDs were formed on sample A, leaving a significant fraction of the InGaAs at its original thickness of 9.6 ML. Because the low-intensity values of $\tau_{F}$ are very close for these two samples we can conclude that capture by 9.6 ML of InGaAs is more efficient than capture by a $6 \mathrm{ML}$ wetting layer, but direct capture by the QDs on sample B makes up for this difference. At higher excitation levels, the $\tau_{F}$ values for sample A are significantly larger than those for sample B, consistent with QDs facilitating carrier relaxation within the InGaAs QL.

Our electron capture times are comparable to those in similar QL systems. (i) Using time resolved transmission, Norris and coworkers published a set of papers studying the carrier dynamics associated with $\mathrm{In}_{0.4} \mathrm{Ga}_{0.6} \mathrm{As} / \mathrm{GaAs}$ QDs (4 closely spaced QD layers, QD base $\sim 14 \mathrm{~nm}$, height $\sim 7 \mathrm{~nm}$, per layer density $\sim 5 \times 10^{10} \mathrm{~cm}^{-2}$, WL thickness $\sim 7 \mathrm{ML}){ }^{3,41-43}$ Their excitation levels are typically less than one carrier per QD. While their work primarily focuses on intralayer dynamics, their modeling of these dynamics suggests a QL capture time of $\sim 0.5 \mathrm{ps},{ }^{43}$ comparable to our capture times. (ii) Liu et al. investigated carrier capture by InAs/ GaAs QDs (QD base $\sim 30 \mathrm{~nm}$, height $\sim 5 \mathrm{~nm}$, density $\sim 4 \times 10^{10} \mathrm{~cm}^{-2}$ ) using Ti:sapphire-laser based pump-probe reflectivity. ${ }^{44}$ Their pump fluence ranged from $\sim 0.008 \mathrm{~mJ} /$ $\mathrm{cm}^{2}$ (comparable to our lowest fluence) to $\sim 0.04 \mathrm{~mJ} / \mathrm{cm}^{2}$ (significantly below our maximum of $\sim 0.3 \mathrm{~mJ} / \mathrm{cm}^{2}$ ). Qualitatively, their reflectivity data are very similar to our data, as is their interpretation of those data. From their fitting, they deduce an electron capture time that varies from $0.25 \mathrm{ps}$ to $0.7 \mathrm{ps}$ as the intensity is increased. They also attribute the increase in capture time versus excitation level to state blocking. (iii) Yarotski et al. also used time resolved reflectivity $(800-875 \mathrm{~nm})$ to study carrier dynamics in InAs/ GaAS QDs (QD base $\sim 40 \mathrm{~nm}$, height $\sim 3 \mathrm{~nm}$, density $\sim 2.7 \times 10^{10} \mathrm{~cm}^{-2}$, WL thickness $\left.\sim 1.5 \mathrm{ML}\right){ }^{45}$ Their pump fluence $\left(0.04 \mathrm{~mJ} / \mathrm{cm}^{2}\right)$ was in the middle of our range of fluences. From their data, they deduce an electron capture time of $0.5 \mathrm{ps}$ for excitation (and probing) with $800 \mathrm{~nm}$ pulses. (iv) Lastly, Li et al. also used pump-probe reflectivity to investigate InAs/GaAs, both below and above the critical thickness $(\sim 1.7 \mathrm{ML})$ for forming InAs QDs. ${ }^{46}$ For an InAs thickness of $1 \mathrm{ML}$ their deduced capture time shows a strong decrease from 4.5 to $0.6 \mathrm{ps}$ as the incident fluence is increased from $\sim 0.001$ to $\sim 0.01 \mathrm{~mJ} / \mathrm{cm}^{2}$. This strong decrease is consistent with cc scattering being responsible for the intensity dependence of their capture times. Apparently their excitation levels are below those where state filling begins to control the capture dynamics. We note that their highest intensity capture time of $0.6 \mathrm{ps}$ is similar to capture times in our study and also that of Yarotski et al ${ }^{45}$ at similar fluences.

\section{SUMMARY}

With time-resolved pump-probe reflectivity, we have investigated carrier dynamics in InGaAs/GaAs QD samples grown using two-stage SK self assembly. Specifically, we have determined electron capture times by these layers, which has provided insight into the influence that the QL morphology has on capture dynamics. Faster capture is facilitated by both a thicker WL and a higher density and/or larger size of the QDs. At high excitation levels state blocking within the QL is observed to hinder the capture process. In conjunction with a diffusion model of carrier transport in the barrier layer, our results are also consistent with ambipolar diffusion as playing the main role in carrier transport in the GaAs barriers near the QL. Inosfar as QL carrier capture is the first step in carrier relaxation and recombination in these systems, further investigation into the connections between morphology and dynamics in QLs fabricated by this novel growth process is warranted.

${ }^{1}$ S. Raymond, S. Fafard, P. Poole, A. Wojs, P. Hawrylak, S. Charbonneau, D. Leonard, R. Leon, P. Petroff, and J. Merz, Phys. Rev. B 54, 11548 (1996).

${ }^{2}$ B. Ohnesorge, M. Albrecht, J. Oshinowo, A. Forchel, and Y. Arakawa, Phys. Rev. B 54, 11532 (1996).

${ }^{3}$ T. Sosnowski, T. Norris, H. Jiang, J. Singh, K. Kamath, and P. Bhattacharya, Phys. Rev. B 57, 9423 (1998).

${ }^{4}$ D. J. Kim, E. A. Everett, and H. Yang, J. Appl. Phys. 101, 106106 (2007).

${ }^{5}$ D. J. Kim and H. Yang, Nanotechnology 19, 475601 (2008).

${ }^{6}$ A. M. Jones, Senior thesis, Brigham Young University (2010).

${ }^{7}$ K. Kamath, P. Bhattacharaya, T. Sosnowski, T. Norris, and J. Philips, Electron. Lett. 32, 1374 (1996).

${ }^{8}$ K. Kamath, N. Chervela, K. K. Linder, T. Sosnowski, H.-T. Jiang, T. Norris, J. Singh, and P. Bhattacharya, Appl. Phys. Lett. 71, 927 (1997).

${ }^{9}$ P. Bhattacharaya, S. Ghosh, S. Pradham, J. Singh, Z.-W. Wu, K. Kim, and T. Norris, IEEE J. Quantum Electron. 39, 952 (2003).

${ }^{10}$ S. Fathpour, M. Holub, S. Chakrabarti, and P. Bhattacharaya, Electron. Lett. 40, 694 (2004).

${ }^{11}$ J. Cederberg, J. Cryst. Growth 307, 44 (2007).

${ }^{12}$ Y. I. Mazur, B. L. Liang, Z. M. Wang, D. Guzun, G. J. Salamo, G. G. Tarasov, and Z. Y. Zhuchenko, J. Appl. Phys. 100, 054316 (2006).

${ }^{13}$ Y. I. Mazur, V. G. Dorogan, J. E. Marega, G. G. Tarasov, D. F. Cesar, V. Lopez-Richard, G. E. Marques, and G. J. Salamo, Appl. Phys. Lett. 94, 123112 (2009).

${ }^{14}$ M. Kudo and T. Mishima, J. Appl. Phys. 78, 1685 (1995).

${ }^{15}$ V. Turck, F. Heinrichsdorff, M. Veit, R. Heitz, M. Grundmann, A. Krost, and D. Bimberg, Appl. Surf. Sci. 123/124, 352 (1998).

${ }^{16}$ G. Sek, P. Poloczek, K. Ryczko, J. Misiewicz, A. Loffler, J. P. Reithmaier, and A. Forchel, J. Appl. Phys. 100, 103529 (2006).

${ }^{17}$ A. J. Sabbah and D. M. Riffe, Phys. Rev. B 66, 165217 (2002).

${ }^{18}$ D. M. Riffe and A. J. Sabbah, Rev. Sci. Instrum. 69, 3099 (1998).

${ }^{19}$ A. V. Kuznetsov, C. S. Kim, and C. J. Stanton, J. Appl. Phys. 80, 5899 (1996).

${ }^{20}$ G. Wang, S. Fafard, D. Leonard, J. E. Bowers, J. L. Merz, and P. M. Petroff, Appl. Phys. Lett. 64, 2815 (1994).

${ }^{21}$ L. Zhang, T. F. Boggess, D. G. Deppe, D. L. Huffaker, O. B. Shchekin, and C. Cao, Appl. Phys. Lett. 76, 1222 (2000). 
${ }^{22}$ S. Bar-Ad, P. Kner, M. V. Marquezini, D. S. Chemla, and K. El Sayed, Phys. Rev. Lett. 77, 3177 (1996).

${ }^{23}$ F. X. Camescasse, A. Alexandrou, D. Hulin, L. Bányai, D. B. Tran Thoai, and H. Haug, Phys. Rev. Lett. 77, 5429 (1996).

${ }^{24}$ B. R. Bennett, R. A. Soref, and J. A. del Alamo, IEEE J. Quantum Electron. 26, 113 (1990).

${ }^{25}$ P. C. Becker, H. L. Fragnito, C. H. B. Cruz, R. L. Fork, J. E. Cunningham, J. E. Henry, and C. V. Shank, Phys. Rev. Lett. 61, 1647 (1988).

${ }^{26}$ W. A. Hügel, M. F. Heinrich, M. Wegener, Q. T. Vu, L. Bányai, and H. Haug, Phys. Rev. Lett. 83, 3313 (1999).

${ }^{27}$ M. T. Portella, J.-Y. Bigot, R. W. Schoenlein, J. E. Cunningham, and C. V. Shank, Appl. Phys. Lett. 60, 2123 (1992).

${ }^{28}$ J. L. Oudar, A. Migus, D. Hulin, G. Grillon, J. Etchepare, and A. Antonetti, Phys. Rev. Lett. 53, 384 (1984).

${ }^{29}$ J. Nunnenkamp, J. H. Collet, J. Klebniczki, J. Kuhl, and K. Ploog, Phys. Rev. B 43, 14047 (1991).

${ }^{30}$ J. H. Collet, S. Hunsche, H. Heesel, and H. Kurz, Phys. Rev. B 50, 10649 (1994).

${ }^{31}$ A. Alexandrou, V. Berger, and D. Hulin, Phys. Rev. B 52, 4654 (1995).

${ }^{32}$ P. Langot, N. Del Fatti, D. Christofilos, R. Tommasi, and F. Vallée, Phys. Rev. B 54, 14487 (1996).

${ }^{33}$ J. Shah, Solid-State Electron. 21, 43 (1978).

${ }^{34}$ A. J. Sabbah and D. M. Riffe, J. Appl. Phys. 88, 6954 (2000).
${ }^{35}$ J. F. Young and H. M. van Driel, Phys. Rev. B 26, 2147 (1982).

${ }^{36}$ G. Bacher, C. Hartmann, H. Schweizer, T. Held, G. Mahler, and H. Nickel, Phys. Rev. B 47, 9545 (1993).

${ }^{37}$ J. H. C. Casey, D. D. Sell, and K. W. Wecht, J. Appl. Phys. 46, 250 (1975).

${ }^{38}$ A. V. Uskov, J. McInerney, F. Adler, H. Schweizer, and M. H. Pilkuhn, Appl. Phys. Lett. 72, 58 (1998).

${ }^{39}$ S. Lutgen, R. A. Kaindl, M. Woerner, T. Elsaesser, A. Hase, H. Künzel, M. Gulia, D. Meglio, and P. Lugli, Phys. Rev. Lett. 77, 3657 (1996).

${ }^{40}$ T. Piwonski, I. O'Driscoll, J. Houlihan, G. Huyet, R. J. Manning, and A. V. Uskov, Appl. Phys. Lett. 90, 122108 (2007).

${ }^{41}$ J. Urayama, T. Norris, J. Singh, and P. Bhattacharya, Phys. Rev. Lett. 86, 4930 (2001)

${ }^{42}$ J. Urayama, T. Norris, H. Jiang, J. Singh, and P. Bhattacharya, Appl. Phys. Lett. 80, 2162 (2002).

${ }^{43}$ J. Urayama, T. Norris, H. Jiang, J. Singh, and P. Bhattacharya, Physica B 316, 74 (2002).

${ }^{44}$ H.-Y. Liu, Z.-M. Meng, Q.-F. Dai, L.-J. Wu, Q. Guo, W. Hu, S.-H. Liu, S. Lan, and T. Yang, J. Appl. Phys. 103, 083121 (2008).

${ }^{45}$ D. A. Yarotski, R. D. Averitt, N. Negre, S. A. Crooker, A. J. Taylor, G. P. Donati, A. Stintz, L. F. Lester, and K. J. Malloy, J. Opt. Soc. Am. B 19, 1480 (2002).

${ }^{46}$ Q. Li, Z. Y. Yu, and W. K. Ge, Solid State Commun. 115, 105 (2000). 